\title{
Nouvelles tendances, vieux problèmes ? Le cas de la réception des « Parcours » à la Villa Méditerranée de Marseille
}

New trends, old problems? The case for reception of the "pathway" at the Ville Méditerranée in Marseille

Nuevas tendencias, antiguos problemas? El caso de la recepción de los "parcours » de la Villa Méditerranée de Marseilla

\section{Snezana Mijailovic et Gloria Romanello}

\section{(2) OpenEdition}

\section{Journals}

Édition électronique

URL : http://journals.openedition.org/iss/456

DOI : $10.4000 /$ iss. 456

ISSN : 2306-4161

Éditeur

ICOM - International Council of Museums

Édition imprimée

Date de publication : 1 décembre 2015

Pagination : 167-184

ISSN : 2309-1290

Référence électronique

Snezana Mijailovic et Gloria Romanello, « Nouvelles tendances, vieux problèmes ? Le cas de la réception des "Parcours » à la Villa Méditerranée de Marseille», ICOFOM Study Series [En ligne], 43b | 2015, mis en ligne le 06 février 2018, consulté le 30 avril 2019. URL : http:// journals.openedition.org/iss/456; DOI : 10.4000/iss.456 


\title{
Nouvelles tendances, vieux problèmes? Le cas de la réception des « Parcours » à la Villa Méditerranée de Marseille
}

\author{
Snezana Mijailovic \\ Aix-Marseille Université - France \\ Gloria Romanello \\ Universitat de Barcelona - Espagne ${ }^{60}$
}

\section{La Villa Méditerranée entre discours officiel et opinion publique}

Au cours de l'année 2013, l'année de Marseille-Provence Capitale Européenne de la Culture, la ville de Marseille s'est vue enrichie par plusieurs lieux culturels permanents ou éphémères. Le Musée des Civilisations de l'Europe et de la Méditerranée (MuCEM), le Frac, le J1, le Château Borély, le Musée d'Histoire de Marseille, entre autres, ont ouvert leurs portes pour la première fois en 2013 ou les ont réouvertes après des travaux de rénovation. Parmi les nouveaux lieux culturels figure également un nouvel espace emblématique de ce renouvellement culturel et urbanistique: la Villa Méditerranée ${ }^{61}$. Inaugurée au mois d'avril 2013, avec ses 10.000 mètres carrés d'espaces utiles, blancs et lumineux et sa présence architecturale remarquable, elle a su rapidement gagner une place d'excellence dans le panorama culturel marseillais, non sans avoir un écho important dans la presse et au sein de l'opinion publique.

La Villa Méditerranée, définie officiellement comme un Centre international pour le dialogue et les échanges en Méditerranée, est un bâtiment d'architecture imposante, " hors norme ", occupant des espaces sur et sous le niveau de la mer et qui se distingue par une avancée en porte-à-faux de 40 mètres « unique en Europe par sa capacité d'accueil du public » (L'architecture, s.d.). Cet élément d'architecture singulier lui vaudra le qualificatif de " plongeoir » et ce sera cette désignation qui circulera dans la ville. Cet édifice met à la disposition des publics trois espaces d'expositions distribués sur trois niveaux, un amphithéâtre de 400 places permettant d'accueillir spectacles, conférences, projections de films, un belvédère, un restaurant - espace café. Selon le discours officiel, la Villa Méditerranée, entièrement conçue, financée et gérée par la Région Provence-Alpes-Côte d'Azur (PACA), repose sur un projet politique et culturel.

Il est politique dans le sens de la coopération décentralisée en aire méditerranéenne, car elle :

$$
\begin{aligned}
& \text { ambitionne d'abord de devenir un lieu d'accueil et de mise } \\
& \text { en contact ouvert à tous les réseaux de coopération. Une } \\
& \text { plate-forme d'échange et de ressources pour ces }
\end{aligned}
$$

\footnotetext{
${ }^{60}$ Cet article s'inscrit dans le cadre du programme de recherche "Publics et pratiques culturelles dans une capitale européenne de la culture - Marseille Provence 2013", Aix-Marseille Université - CNRS, LAMES UMR 7305 (avec le soutien du ministère de la Culture et de la Communication, de la MSH Paris Nord, de la région PACA).

${ }^{61}$ L'ensemble architectural de la Villa Méditerranée a été imaginé par l'architecte italien Stefano Boeri, lors du concours public de la région PACA de 2004. Sa construction a démarré en 2012 pour un coût total estimé à 70 millions d'euros.
} 
spécialistes d'organismes internationaux, de «think tanks ", de collectivités locales et territoriales, de centres de recherches divers ou de milieux économiques ( Le projet », s.d.).

Il est culturel, parce que l'offre culturelle proposée est basée sur la création de parcours d'exposition, soit permanents ${ }^{62}$ soit temporaires, ainsi que sur une intense programmation artistique et culturelle le soir $^{63}$. Les objectifs stratégiques de cette nouvelle institution citoyenne et culturelle sont explicités dans le dossier de présentation. II s'agit de :

\begin{abstract}
s'emparer des grands enjeux de la Méditerranée contemporaine, y aborder les questions actuelles et de prospectives, telle est l'ambition de la Villa Méditerranée. Économie, environnement, urbanisme, (r)évolutions politiques [...] Ce territoire entretient depuis longtemps les préjugés et les idées toutes faites. La Villa Méditerranée s'est donnée pour mission - aussi exigeante que passionnante - de les dépasser (Dossier de présentation, 2013).
\end{abstract}

Contrairement à ce que l'on peut entendre ou supposer du fait de l'année de son inauguration, la Villa Méditerranée n'a pas été conçue à l'occasion de l'obtention du titre de Capitale européenne de la culture décerné à Marseille en 2013 ${ }^{64}$. En effet, l'idée de sa construction date de l'année 1999 et du premier mandat de Michel Vauzelle (président de la région PACA) qui est à l'origine de ce projet $^{65}$. Mais la concordance de calendrier ne va pas manquer de créer un amalgame entre son ouverture et Marseille-Provence 2013 et semble même avoir eu un certain poids dans la construction de «l'horizon d'attente » (Jauss, 1990) des publics. La Villa Méditerranée a très rapidement soulevé une polémique auprès des Marseillais, comme le résume le journal Zibeline ${ }^{66}$ :

en ces temps de grands chantiers culturels arrêtés partout en France par le nouveau Ministère de la Culture, de restriction sur tous les budgets de fonctionnement et de peur panique devant les investissements jugés onéreux des collectivités, la naissance de la Villa Méditerranée fait jaser les poujadistes, mais pas seulement : chantier pharaonique, doublant le MuCEM, né d'un caprice régalien de Michel Vauzelle, au contenu culturel vague sinon vide... les reproches sont légion, y compris parmi ceux qui défendent habituellement la pertinence économique et humaine des investissements culturels (Freschel \& Cloarec, 2012).

La Villa Méditerranée, si elle a séduit les uns, a aussi déplu aux autres, provoquant parfois des réactions négatives très critiques, et la presse a de ce point de vue souvent joué un rôle significatif. Le coût élevé en termes économiques, la question de l'utilité et de la légitimité de cette nouvelle institution ont été les sujets les plus

\footnotetext{
${ }^{62}$ A la Villa Méditerranée les parcours de visite appelés permanents sont, en effet, d'une durée de trois ans.

${ }^{63}$ Projections et festivals de cinéma, concerts, théâtre, spectacles de danse, etc.

${ }^{64}$ Comme tenait à nous le préciser l'un des agents d'accueil lors d'un des entretiens que nous avons conduits dans le cadre de cette recherche.

${ }^{65}$ On notera ici que le projet de la Villa s'inscrit dans la même temporalité que celui du MuCEM.

${ }_{66}^{6}$ Zibeline est un journal qui traite des événements culturels à Marseille et en région PACA.
} 
fréquemment abordés dans le débat. Ces quelques citations làdesous très parlantes et parfois provocatrices en témoignent :

un chantier à 70 millions d'euros. Le plus onéreux des équipements bâtis dans le sillage de Marseille-Provence 2013 est aussi celui dont l'utilité est la plus contestée (Piscopo-Reguieg, 2013);

voici la villa la plus chère de la région Provence-AlpesCôte-D'Azur: 70 millions payés rubis sur l'ongle par les gentils contribuables (Oberson, 2013);

à côté de l'incontestable réussite du MuCEM, il y a la contestée Villa Méditerranée. Dont l'architecte est en procès pour plagiat ${ }^{67}$, qui se fait allumer dans la presse pour son coût, son esthétique, son "absence de projet". Tout cela est fort peu mesuré : initiée et financée dans un temps où le MuCEM était en panne, la Villa Méditerranée a su trouver des complémentarités culturelles (Freschel, 2013).

Et, en effet, un dernier élément de cadrage qu'on ne peut passer sous silence, qui est d'ailleurs évoqué dans cette dernière citation, renvoie à cette inévitable relation de voisinage de la Villa Méditerranée avec le musée «iconique » du MuCEM (Buslacchi, Girel \& Maisetti, 2015). Avec cet autre équipement culturel emblématique de l'année 2013, la Villa ne partage pas seulement un espace physique sur l'esplanade réaménagée et accessible du $\mathrm{J} 4^{68}$, mais bien aussi un espace épistémologique : c'est de ce point de vue que le défi comparatif entre les deux institutions culturelles semble être inévitable.

\section{Les « parcours », la marque de fabrique}

L'appareil sémantique utilisé dans le discours officiel de présentation de la Villa Méditerranée nous livre également quelques éléments interprétatifs sur les apports culturels, sur les choix narratifs en termes d'offres culturelles et finalement sur les publics auxquels elle s'adresse. La Villa semble bien d'abord se consacrer au grand public $^{69}$ :

l'originalité du projet Villa Méditerranée tient ainsi à cette capacité à rendre accessibles au grand public des thématiques traditionnellement réservées aux chercheurs et aux experts (Dossier de présentation, 2013).

Ces thématiques sont matérialisées et adressées aux visiteurs par la création des "parcours » d'exposition, eux-mêmes complétés par le reste de la programmation («Le projet »). Les «parcours » constituent le cœur de la proposition culturelle de la Villa : considérés par la Villa comme sa marque de fabrique, ils sont conçus et réalisés par des « narrateurs » qui, en tant qu'artistes, cinéastes, écrivains, metteurs en scène, « exposent la parole des citoyens et des scientifiques » (« Le projet») par le biais d'une mise en scène construite et élaborée sur de l'audiovisuel.

\footnotetext{
${ }^{67}$ Pour une synthèse cf. Savane, 2013.

${ }^{68}$ Sur le J4 et son histoire cf Jordi, 2013.

69 «Du Marseillais au croisiériste, du lycéen au diplomate, du touriste à la famille habitant le quartier du Panier tout proche... chacun trouvera un parcours ou une programmation à son goût. C'est le premier défi de la Villa Méditerranée : mêler grand public et acteurs de la coopération méditerranéenne » (Dossier de présentation, 2013).
} 
Concrètement, après quelques événements ponctuels ${ }^{70}$ et l'organisation de visites architecturales, la Villa Méditerranée a définitivement ouvert ses portes aux publics avec l'inauguration des deux premiers parcours d'exposition : Plus loin que l'horizon et 2031 en Méditerranée, nos futurs ! Le premier est un parcours situé au sous-sol de la Villa, créé par Bruno Ulmer, réalisateur de documentaires. Son statut est celui d'un parcours «permanent »; il est prévu qu'il soit actualisé à plusieurs reprises pendant sa durée (de trois ans). Ce parcours porte sur les mobilités en Méditerranée : mobilités humaines (tourisme, exil, migrations, avec une attention particulière sur les immigrés clandestins) et mobilités marchandes (entre autres, la route de la fraise récoltée en Espagne, la marchandise qui voyage d'Istanbul à Alger); il est conçu avec une évidente volonté de sensibilisation aux enjeux sociaux touchant ce territoire. Plus loin que l'horizon commence de prime abord sur les longs et larges escaliers en forme de spirale qui mènent du niveau de l'entrée au niveau -2 de la Villa. Sur un grand mur, défilent des mots suggestifs en plusieurs langues méditerranéennes, accompagnés par des sons de cloches des églises, des bêlements, des chants orthodoxes grecs, entre autres. L'effet recherché est celui d'une surprise et d'une immersion progressive, à la fois émotive et physique, des visiteurs dans l'exposition. La suite du parcours est composée de plusieurs espaces élaborés notamment sur de l'audiovisuel, dédiés chacun à un récit de mobilité. La scénographie plonge les visiteurs physiquement dans ces réalités et leur permet de circuler librement d'une histoire à l'autre. A l'issue du parcours, ils sont invités à participer activement en répondant par écrit à la question "Les mobilités en Méditerranée, vous en dites quoi ? " en collant des post-it jaunes laissés à leur disposition sur un grand mur à côté de l'entrée-sortie.

Le deuxième parcours, 2031 en Méditerranée, nos futurs !, invitait les visiteurs dans une installation temporaire qui a occupé l'espace d'exposition du porte-à-faux du mois de juin au mois de septembre 2013. Ce parcours a été créé par Régis Sauder cinéaste documentariste, qui a choisi de donner la parole à des jeunes de quatre villes du pourtour méditerranéen (Izmir, Tunis, Beyrouth, Marseille) à travers des ateliers participatifs, afin de transmettre leurs espoirs, leurs angoisses, leurs rêves, enfin, leurs visions de l'avenir en Méditerranée dans vingt ans. Ce parcours était articulé autour de quatre espaces thématiques, chacun consacré à un sujet spécifique : identité, environnement, conflits, slogans et issus d'un travail d'expression libre. Chaque espace était construit sur le même modèle, celui d'un box, d'une cellule, une sorte de "boîtes à images ». Les images étaient projetées sur les quatre faces internes de ces quatre cellules liées entre elles par des fils électriques, sorte de fils conducteurs, tandis que les faces externes avaient été couvertes par les représentations de l'avenir proposées par les jeunes et transformées en dessins par un dessinateur professionnel. Ici aussi les visiteurs étaient invités à participer : par le biais d'une table à dessins, il leur était proposé de partager leurs propres visions du futur en Méditerranée ; ils pouvaient faire des dessins ou écrire des slogans qui étaient alors projetés en direct sur un mur et donc rendus immédiatement publics par le biais d'un dispositif vidéo.

\footnotetext{
${ }^{70}$ Forum Libération, Rencontres internationales des cinémas arabes, entre autres.
} 


\section{Un « projet audacieux » entre glissements terminologiques et choix programmatiques}

L'effort de ce nouvel établissement public semble donc être marqué depuis le début par une volonté réformatrice et pionnière, soit au regard des contenus proposés, soit dans la façon dont ces contenus vont être communiqués aux publics. En effet, déjà dans le discours institutionnel initial, l'exposition se fait " parcours », le commissaire « narrateur », le médiateur " agent d'accueil » et finalement le visiteur devient "acteur " ${ }^{71}$. Concernant la démarche de la Villa Méditerranée, Bruno Ulmer ${ }^{72}$ souligne qu'il s'agit de proposer un cheminement dans lequel se trouve : en amont tout ce qui se passe dans la Villa et nourrit la réflexion (les projets de coopération avec les pays méditerranéens par exemple) et en aval l'offre culturelle (le théâtre, les projections de films, les concerts, etc.), les parcours se trouvant quant à eux au milieu et n'étant qu'une étape pour cheminer vers l'un et/ou l'autre. Du point de vue de la médiation, à l'intérieur et tout au long des parcours, la direction de la Villa Méditerranée a délibérément préféré éviter la présence des médiateurs pour laisser les visiteurs libres

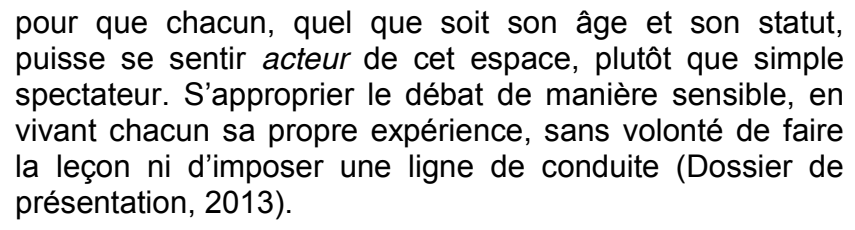

Néanmoins, des " agents d'accueil » sont présents à l'extérieur des parcours, leur rôle étant d'être en contact direct avec les visiteurs pour recueillir leurs réactions concernant les " parcours" et les services.

Arrivées à ce point de notre présentation, nous devons maintenant nous interroger : quels sont donc les effets de ce " projet audacieux " ( "Le projet ») ? Quelles sont les conséquences de ces glissements (terminologiques et en termes de dispositifs) dans l'expérience quotidienne de la Villa Méditerranée ? Comment s'intègre ce supposé changement programmatique dans les différents champs de l'offre culturelle et surtout dans la relation avec les publics ? Quels sont en définitive leurs retours? Quels messages, appréciations, impressions, expriment-ils et que reflètent-ils ?

II faut ici d'abord insister sur l'évolution du rôle des publics qui de visiteurs-spectateurs passifs deviennent des acteurs engagés dans le processus même de création des apports culturels de l'institution. Dans un même temps et paradoxalement, il faut prendre en considération que les auteurs-créateurs des parcours sont amenés à gérer la communication en devenant "démiurges " d'une narration qui pourtant ne leur appartient qu'en partie. Cela étant dit, nous observons que la déclaration d'intention vigoureuse en faveur d'un renouvellement narratif de l'offre culturelle (qui figure comme l'un des enjeux majeurs) non seulement opère une modification dans le choix terminologique, mais entraîne également des conséquences au niveau des fonctions que la nouvelle institution culturelle marseillaise s'autorise à exercer. Se pose alors la question de l'adéquation

\footnotetext{
${ }^{71}$ Les propos sur ce glissement terminologique sont recueillis durant les entretiens avec les chargés de productions et des publics et avec les agents d'accueil.

${ }^{72}$ Nous avons recueilli ces propos lors de la présentation du parcours Plus loin que I'horizon par Bruno Ulmer aux agents d'accueil.
} 
formelle des contenus proposés avec les contraintes rhétoriques que nous venons d'exposer. Les problématiques envisagées relèvent de ce fait de différents - mais synchroniques - niveaux d'explication et touchent les domaines interdépendants suivants :

La relation avec les publics. $\mathrm{Si}$, comme nous venons de voir, la définition du public est effectivement touchée par ce changement sémantique, est-il possible d'observer des changements significatifs dans l'approche des publics face aux différents champs de l'offre (apports culturels, aides à la visite, confort et services) et à leurs sous-champs? Sur le plan du renouvellement formel des politiques des publics des institutions culturelles (Ballé \& Poulot, 1995; Davallon, 1992), dans quelle trajectoire se trouve la Villa ? S'inscritelle dans le débat de la muséologie participative (Chaumier, 2007; Simon, 2010), comme semblent nous suggérer les discours officiels ? Jusqu'à quel point ? Quels sont les effets et les résultats de ses choix stratégiques en matière de politique des publics?

La définition de la mission et des fonctions. Quelle est l'identité de la Villa ? En quoi consiste sa raison d'être ? S'agit-t-il d'une nouveauté hybride (Mairesse, 2010) pour sa capacité d'intégrer plusieurs aspects en termes de politique culturelle et de gouvernance d'établissement? Peut-être faut-il réfléchir à une définition, en creux, en négatif et la définir par ce qu'elle n'est pas (elle n'est pas un musée, pas un palais des congrès, etc.). Ces discours nous conduisent à considérer aussi le positionnement de cet établissement dans la ville de Marseille, en relation avec les autres équipements culturels dans la ville dans le contexte des politiques culturelles locales (Davallon, 1997; Poirrier, 1996; Tobelem \& Benito, 2001).

\section{Itinéraire méthodologique et approche interprétative}

L'itinéraire méthodologique utilisé comporte deux phases successives, mais qui sont rapprochées dans la présentation des résultats. Dans une première phase, nous avons effectué une recherche exploratoire à travers la lecture et l'analyse de la documentation officielle, des articles de presse et ce afin d'identifier les problématiques dignes d'intérêt aux yeux du chercheur. Cette phase d'instruction de l'étude a été complétée par un corpus d'entretiens informels et semi-directifs conduits avec les acteurs protagonistes de la vie de l'institution : les personnes travaillant dans les équipes de production et de relations publiques et internationales, les agents d'accueil ou encore les visiteurs. Ce corpus d'entretiens nous a permis d'avoir accès à des informations de nature qualitative, et notamment d'avoir un retour sur les expériences directes ou les opinions plus personnelles face à l'expérience quotidienne au sein de la Villa Méditerranée. Les entretiens semi-directifs avec les agents d'accueil, considérés comme les récepteurs directs des réactions des publics, ont été particulièrement significatifs. Ils nous ont permis de comprendre et reconstruire un premier cadre interprétatif de références sur les comportements des visiteurs et à partir de leurs propres expériences de visites. À travers l'usage combiné des entretiens, de l'analyse documentaire et d'une observation participante $^{73}$ auprès des publics de la Villa, nous avons alors pu

\footnotetext{
${ }^{73}$ Les observations ont été effectuées pendant la période du mois de juin au mois de novembre 2013. Le début de terrain a été déterminé par l'ouverture de deux premiers parcours et la fin par la saturation des éléments observés. Au cours des observations, notre attention a été centrée surtout sur les comportements des visiteurs dans les
} 
construire une sorte de carte conceptuelle des thématiques qui permettait d'appréhender la réception des parcours ainsi que de la perception globale de la Villa Méditerranée en tant que nouvelle institution culturelle marseillaise.

Au cours de l'analyse qui suit, nous proposons d'adopter une mise en perspective critique par la mise en relations de deux points de vue : (1) celui des discours programmatiques, les attentes et les offres institutionnelles, (2) celui de l'évidence des réceptions par les publics, à travers la mise en place d'une comparaison systématique de ces deux axes épistémologiques. Rappelons ici que la deuxième phase de recherche a intéressé plus directement les publics de la Villa Méditerranée, ceux auxquels l'institution même s'adresse en tant qu' " acteurs » des "parcours » de visite, faisant valoir ainsi une certaine attente participative à l'égard de ses publics, appelés promptement à agir sur le plan de l'expérience personnelle (Le Marec, 2007). Si l'on considère l'évaluation comme une démarche d'observation liée à l'interprétation des effets d'une action (DufresneTassé, 2002), on comprend bien alors que saisir la perspective de la perception ainsi que les degrés de satisfaction des visiteursacteurs devient un passage obligé et indispensable (Eidelman, Roustan \& Goldstein 2007 ; Tobelem, 2003, 2010) dans la mise en perspective des politiques de l'établissement. Pour ces raisons, nous avons choisi de concentrer aussi notre attention critique sur le Livre d'or, un instrument souvent considéré comme partiel ou superficiel, dont l'usage est souvent discuté, mais qui « offre un incomparable point d'appui pour permettre l'échange vertical des points de vue des visiteurs vers l'établissement» (Béra \& Paris, 2007). Le Livre d'or $^{74}$ nous permet d'obtenir des données qualitatives tangibles qui nous permettent d'évaluer les effets produits par les choix conçus par la Villa Méditerranée. Conscientes des limites de représentativité que cette démarche d'investigation comporte ${ }^{75}$, les auteures ont eu pour but de saisir la gamme des signifiés possibles pour répondre aux objectifs de cette recherche. En faisant cela, cette étude vise à franchir les limites des définitions fermées au service d'une perspective critique qui observe la relation entre les attentes institutionnelles (telles qu'elles ressortent dans le contexte de la communication officielle, de la réponse de la presse marseillaise et finalement à travers la voix interne du staff interviewé) et l'évidence de la réception des «parcours » proposés grâce aux contributions spontanées des visiteurs sur le Livre d'Or de la Villa ou sur les post-it mis à disposition lors du parcours permanent, qui, comme il était assez facile de le prévoir, sont devenus aussi un lieu de réflexion et de questionnement sur la Villa Méditerranée en tant que telle.

\section{La réception des "parcours": les visiteurs face aux attentes}

L'analyse des contenus explicites et implicites tirés des commentaires du Livre d'or nous renvoie une image fiable de la diversité des avis des publics, et les commentaires constituent des indicateurs des

parcours, leurs logiques de circulation et leurs réactions aux contenus et mises en scène des parcours.

${ }^{74}$ Rendu disponible (conjointement avec une sélection des post-it du parcours Plus loin que l'horizon) pour les finalités de cette recherche grâce à la disponibilité du staff de la Villa Méditerranée.

${ }^{75} \mathrm{Au}$-delà de difficultés méthodologiques indéniables, puisque "le livre d'or confronte plus que tout autre l'interprétant à la précarité de ses interprétation [...] Le livre d'or n'offre donc ni la représentativité du quantitatif, ni la finesse d'analyse du qualitatif » (Béra \& Paris, 2008). 
tendances majoritaires qui tracent la voie à la compréhension de la réception de l'offre culturelle de l'établissement et de sa mission.

Tout en déclarant les différences d'approche avec les enquêtes de satisfaction ou de perception au sens classique des termes, laisser un commentaire sur un Livre d'or demeure un acte démonstratif fort qui mobilise la volonté manifeste du visiteur de laisser une trace de son passage physique et intellectuel, sa motivation principale étant " de nouer relation avec l'établissement culturel, de l'aider à définir sa place dans la société » (Béra \& Paris, 2007). Ainsi, pour comprendre les effets de la (prétendue ?) volonté réformatrice de la Villa Méditerranée dans la définition de ses stratégies, nous allons observer l'expérience quotidienne de réception des " parcours " par les visiteurs à travers les commentaires du Livre d'or et les post-it, car ce sont :

de tous les outils à disposition des établissements culturels
pour identifier leurs visiteurs et ajuster leurs stratégies,
ceux qui restituent le mieux la richesse de leur imaginaire,
l'ampleur de leurs motivations et la diversité des liens
noués avec l'établissement (Béra \& Paris, 2007).

En adaptant nos paramètres d'observation aux lignes directrices proposées récemment par le Département de la Politique des publics du Ministère de la Culture (Eidelman \& Jonchery, 2013), nous proposons d'approfondir l'expérience de visite des "parcours " à travers les opinions exprimées pour les personnes qui les énoncent selon les thématiques qui suivent.

\section{Les champs de l'offre et la satisfaction des publics}

Dans l'interprétation de la satisfaction des publics des "parcours" de la Villa Méditerranée, l'enrichissement personnel - à travers les contenus culturels et sociaux présentés - semble être le côté le plus apprécié. Mais la satisfaction détenue est la plus forte quand l'appréciation s'accompagne de l'expression des sentiments éprouvés lors de la visite. Le désir de laisser un commentaire semble être déclenché par une forte émotion ; l'expression de la satisfaction est alors une conséquence de cette expérience bouleversante provoquée par les contenus présentés dans le "parcours " (Plus loin que l'horizon en prévalence statistique sur $2031^{76}$ ), parcours qu'il faut aborder impérativement « avec la tête et avec le cœur » :

Ce parcours est intelligent parce qu'il est réaliste, sans complaisance; Mais je sors d'ici enthousiaste et remplie d'espoir ;

Superbe, très pédagogique et surtout très émotionnelle ;

Extraordinaire exposition, courageuse, qui ne peut que toucher les méditerranéens que nous sommes et convoque la solidarité ;

Témoignages touchants ;

Endroit qui nous permet de toucher des doigts des problématiques qui nous touchent et qui sont à côté de nous! ;

On ressort bousculé ;

On s'en prend plein la gueule.

Les commentaires relatifs à la satisfaction esthétique sont ceux où l'on retrouve le plus de déceptions ; ils sont présents, même si moins représentatifs. Dans tous les cas, la beauté semble nouer une

\footnotetext{
${ }^{76}$ Le fait que le parcours temporaire soit payant et le permanent "d'entrée libre" a eu comme conséquence la répartition des visites entre parcours payant/gratuit de 20/80, soit 21.259/95.994 visites pour la durée du parcours temporaire (source: documentation interne de la Villa Méditerranée).
} 
relation stricte avec la capacité émotive de la scénographie et sa capacité de " faire réfléchir » :

Une scénographie extrêmement qualifiée ;

J'aime l'ambiance "immersion totale".

En ce qui concerne les aides à la visite, l'accueil et le personnel recueillent la satisfaction de la plupart des visitateurs (« La réception est extrêmement accueillante et motivante »), tandis qu'un certain manque de communication et de médiation humaine est toutefois souvent signalé, mais il n'empêche pas l'appréciation globale de l'expérience de visite (nombreux sont les commentaires " partagés » entre la satisfaction enthousiaste par rapport aux contenus et la déception eu égard aux aides à la visite). Cependant, il y a aussi ceux qui comprennent et approuvent le choix des " narrateurs " de ne pas intervenir davantage dans l'interprétation: "Continuez à donner la liberté à chacun! ».

Peu de remarques ont été faites au sujet du confort et des services ; la plupart de ceux qui les ont faites dénoncent l'absence de sièges, le manque de lumière, la trop haute fréquentation en relation avec les espaces réduits, mais ce qui revient est surtout lié à la politique des prix (Eidelman \& Céroux, 2009): la gratuité de l'un des parcours face au caractère payant de l'autre induit de fait une comparaison très sensible entre les deux parcours et donc, les critiques (de manière semblable aux contenus et aux services) se multiplient en fonction probablement de l'augmentation des attentes. Pour le dire plus simplement, le fait de payer implique de la part des visiteurs des attentes plus exigeantes et qui réfèrent à leurs expériences de visites d'autres lieux (musées par exemple). Du même style, mais en touchant des critères de pertinence et d'utilité publique, on note la critique du "gaspillage d'argent public »: elle ne concerne qu'en partie les choix curatoriaux et s'intéresse plutôt à la perception d'ensemble de l'établissement.

Le rapport aux attentes, les perceptions, les motivations de la visite

Parcourir l'ample éventail des ressentis, des perceptions, des projections axiologiques des visiteurs a été notre principal souci interprétatif. La perception des parcours de visite et l'expression des ressentis semblent être loin de faire l'unanimité et témoignent de la diversité des ressources auxquelles les visiteurs font référence au moment d'établir une échelle des priorités sur laquelle mesurer leurs expériences. On reconnaît qu'en général l'univers des locutions positives l'emporte sur l'expression des perplexités et des critiques; ces réponses positives touchent en préférence l'horizon des valeurs et nous parlent de la capacité à susciter de fortes émotions d'étonnement positif et de bouleversement émotif. Au contraire, quand il s'agit de présenter des points négatifs, la déception concerne les contenus intellectuels et la sphère de la connaissance et de l'apprentissage, côté qui semble être l'objet des plus hautes attentes selon les visiteurs les plus exigeants. 


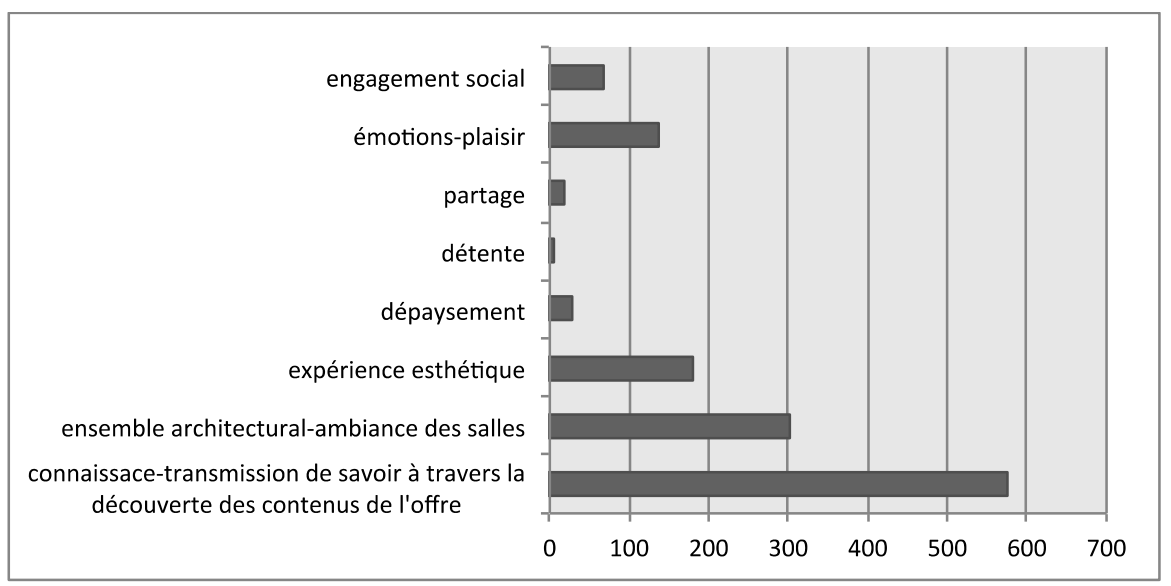

Source : élaboration propre à partir des données recueillies

L'analyse de l'univers symbolique des motivations de la visite entre les différents commentaires est l'indice d'un certain degré d'utilité perçue (indicative, à cet égard, est la fréquence de l'usage de l'adjectif "utile»). S'il est pourtant vrai que le motif cognitif active l'action de visite et déclenche l'expression d'un commentaire écrit, nous remarquons la présence aussi des ressentis et motifs axiologiques. Parmi ces derniers, domine la présence d'un trait très représentatif des visiteurs de ces "parcours " qui s'ajoute à ceux plus structurants de toute expérience de visite (Eidelman \& Jonchery, 2013) et qui semble décrire au mieux l'identité de notre visiteur, l'engagement social, qui revient assez régulièrement entre les commentaires :

\section{Bravo pour la déclaration de toutes les injustices dans le musée ; \\ Excellent pour un nouveau regard sur tous les peuples de la Méditerranée ; \\ Quel qu'ils soient pauvres ou riches, ils sont appelés à vivre solidaires dans un esprit de solidarité universelle. Grand merci pour cet appel!}

\section{La satisfaction par rapport aux caractéristiques de la prescription}

S'il a été possible de reconduire les contenus explicites et implicites des commentaires au rang des ressentis et des motivations de la venue, nous adoptons également la proposition de construire des indicateurs synthétiques de la satisfaction en fonction des caractéristiques identifiables de la prescription. Après l'opérationnalisation et la mise en relation des données issues des commentaires, selon la formulation d'Eidelman et Jonchery (2013), notre catégorisation des résultats nous renvoit une image assez polarisée de la propension à la prescription :

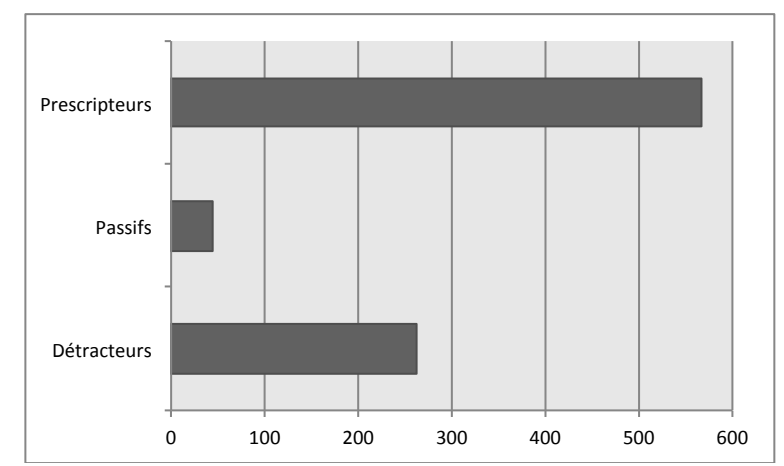

Source : élaboration propre à partir des données recueillies 


\section{Architecture et ambiance}

Il est évident, et c'était prévisible, que cet aspect de la visite ne passe pas inaperçu. Appréciée ou détestée, la relevance de l'architecture suscite un débat très vif aussi dans les pages du Livre d'or et sur les post-it et mérite une mention à part. Dépassant les limites de la satisfaction en positif ou en négatif, la présence physique et concrète de la Villa Méditerranée gagne sa place de protagoniste, même quand c'est le rapport contenus/contenant, voire le vide, qui retient le plus l'attention des visiteurs : " Le bâtiment est superbe, mais, hélas, un beau squelette ».

En conclusion, très appréciés ou extrêmement critiqués, les "parcours » ainsi que la globalité de l'établissement de la Villa, ne laissent pas indifférent. Au contraire, ils provoquent une prise de position éclatante, souvent vive, qui laisse peu ou pas de place aux positions moyennes et qui semble être une conséquence directe de la participation émotionnelle et de l'engagement personnel des « acteurs » que la visite implique. Les stratégies mises en place par l'institution sont donc loin de recevoir une appréciation unanime, unilatérale; pourtant, elles se révèlent habiles à capturer leurs audiences par le biais des émotions frappantes, même si c'est avec de larges zones d'ombre, comme le démontre la présence marquée d'un public de détracteurs.

\section{«On passe notre temps à dire ce que l'on n'est pas » : les fonctions et l'identité de la Villa Méditerranée aux yeux de ses publics}

A l'issue du point précédent, revenons au projet, pour en mesurer les effets.

Depuis sa conception en 1999, la Villa Méditerranée a changé de nom $^{77}$ et son projet a également beaucoup évolué (Gilles, 2013), circonstances qui apparemment n'ont pas facilité l'identification de sa mission. Quelques jours après son inauguration officielle, Télérama écrivait: "La Villa Méditerranée est en quête de sens. Elle se cherche une place, une raison d'être", (Piscopo-Reguieg, 2013). L'article reflétait les rumeurs qui existaient déjà au sein de la population locale et qui laissait entendre que, même au moment de l'ouverture, le discours officiel manquait de précision. Avoir observé comment les visiteurs réagissaient aux "parcours » nous permet d'aborder ici le thème de la réception de la Villa Méditerranée en tant que telle. Pour mieux cerner certains aspects, nous étendrons le corpus de nos données aussi aux entretiens réalisés auprès des visiteurs.

L'un des éléments qui a retenu notre attention parmi les données renvoie à la diversité des représentations que les visiteurs se sont construit de la Villa. Le manque de clarté dans le discours officiel ainsi que le manque de communication appropriée concernant ses contenus paraissent contraindre les visiteurs à mobiliser leurs références personnelles ou l'expérience effective de visite dans la tentative de saisir les missions de la Villa. Dans ce sens, on constate l'existence de définitions des fonctions qui divergent de la présentation institutionnelle et qui revêtent des formes différentes :

\footnotetext{
77 Son premier nom était CeReM (Centre Régional de la Méditerranée). Voir: Leforestier, 2013.
} 
Présenter la culture méditerranéenne avec un format vidéo très contemporain ;

Faire découvrir la Méditerranée et les gens autour ;

Être un lieu de conférences et colloques.

Si l'on compare ces affirmations avec la présentation officielle, les fonctions de la Villa perçues chez les visiteurs peuvent être caractérisées comme incomplètes et diversifiées à cause de l'impossibilité de leur part de saisir la pluralité des missions que la Villa veut s'attribuer, cette impossibilité semblant être le résultat de l'absence de moyens qu'elle met à leur disposition pour se faire comprendre.

Aussi, du point de vue de la Villa, ces perceptions peuvent être considérées comme incorrectes, ne correspondant pas à l'appareil sémantique qu'elle a adopté. En effet, on observe que, leur visite à la Villa ayant consisté en une visite de "parcours », certains visiteurs pensent que sa fonction principale est d'être " un lieu d'exposition » voire «un musée » et ce malgré le positionnement institutionnel qui insiste pour présenter «les parcours » comme différents « des expositions » et la Villa comme n'étant pas un bâtiment culturel. Ici, ce sont l'expérience directe de la visite et l'expérience personnelle par rapport aux pratiques culturelles qui l'emportent chez les visiteurs sur la volonté innovatrice de la Villa. La difficulté rencontrée par les publics à saisir ses fonctions et sa raison d'être peut aboutir à l'absence d'identification malgré la(les) visite(s) effectuée(s) comme l'illustre cet extrait d'entretien avec une personne s'étant rendue deux fois à la Villa : "Je savais pas trop quelle était sa (fonction)... d'ailleurs, je sais toujours pas".

De leur côté, les expressions de la satisfaction et de l'insatisfaction dans le Livre d'or et sur les post-it concernant les "parcours » nous indiquent indirectement les horizons d'attentes et leur rapport avec les fonctions perçues. Certains visiteurs enthousiastes attribuent à la Villa une fonction pédagogique et instructive contrairement à sa volonté de "ne pas faire la leçon » (Dossier de présentation, 2013). La forte insatisfaction de ceux qui critiquent la sous-représentation des pays africains de la Méditerranée dans les parcours nous parle des fonctions qui sont attribuées à la Villa en raison de son nom (« Je croyais que la Méditerranée avait 2 rives. Ici, n'apparaît qu'une seule rive et je trouve cela très regrettable »). Se fixant sur ce qui n'est pas là, ces visiteurs jugent «ratée » sa mission de représenter l'aire géographique dont elle porte le nom.

Une autre tendance parmi les réactions négatives, déjà évoquée, porte sur la question du gaspillage de l'argent public. Ce sujet est soulevé également chez les visiteurs ayant apprécié les parcours. Cela renvoie à la fameuse question du doublon, doublon avec le MuCEM, mais aussi probablement avec les autres institutions marseillaises qui sont considérées comme les lieux habituels (donc plus légitimes) pour organiser les conférences et séminaires. Dans un même temps, ces tendances de critiques négatives nous dévoilent l'existence des attentes qui ne sont pas remplies en reflétant une perception de la Villa qui est loin d'être idéale.

Enfin, les visiteurs semblent reconnaître une autre fonction à la Villa : celle d'agent contributeur au rayonnement culturel de la ville. Cette fonction se présente comme particulièrement significative dans le contexte de MP2013 et témoigne de l'existence de plusieurs facettes des publics marseillais. A côté d'un Marseille détracteur préoccupé par la destination de l'argent public (et donc opposé à la construction de la Villa), à côté aussi de l'autre Marseille, également détracteur, 
boycottant MP2013 en le considérant élitiste, les commentaires prouvent l'existence d'un troisième Marseille, qui désire un changement d'image et un nouveau positionnement sur le marché touristique. La répétition de l'adjectif « enfin » nous dévoile l'existence des attentes de longue date le concernant:

\author{
Soyons enfin fiers !!! \\ Enfin! Marseille a quelque chose de beau à nous faire \\ partager. Merci.
}

Somme toute, la nouveauté du lieu semble entraîner avec elle l'absence des représentations construites. La parole des visiteurs n'étant pas encore visible, les publics sont amenés à bricoler euxmêmes leurs représentations en puisant dans leurs propres références, d'où l'existence d'une diversité des représentations qui la plupart du temps n'arrivent à définir la Villa qu'en partie. Cela met à jour un décalage entre la volonté du lieu d'être plusieurs choses à la fois et la façon dont il le communique aux publics.

\title{
"Mais, on est pas au MuCEM ? » ou Le MuCEM, un voisin gênant ?
}

Les fonctions attribuées à la Villa Méditerranée semblent nous indiquer une identité perçue comme proche de celle des musées des civilisations (Eidelman \& Jonchery, 2013) d'où aussi le rapprochement obligé avec le MuCEM. La proximité physique et épistémologique a soulevé, dans l'opinion publique, la question du doublon. Comment justifier que ces deux monuments soient consacrés à des thématiques similaires et situés à quelques mètres l'un de l'autre ? On notera que, dans ce questionnement, la légitimité du MuCEM n'a pas été remise en cause, s'agissant d'un projet d'envergure nationale. En effet, cette ambition affichée par la Villa de «traiter des questions actuelles et de prospectives " (Dossier de présentation, 2013) lui a servi d'élément de démarcation par rapport au MuCEM, qui, en tant que musée, serait censé s'occuper de I'histoire : " Le MuCEM, c'est un musée, c'est donc l'histoire ; la Villa est le lieu qui regardera vers l'avenir ${ }^{78}$ (Piscopo-Reguieg, 2013). Mais ce découpage dans les discours d'intention pose problème au seul regard de la programmation des deux institutions. Premièrement, le MuCEM est loin de limiter l'étendue de ses thématiques au passé tant dans le contenu de ses expositions que dans le reste de l'offre qui les accompagne. Deuxièmement, la Villa elle-même n'est pas étrangère aux thématiques liées au passé méditerranéen, dont témoigne son nouveau parcours permanent, Échelles des temps, qui retrace l'histoire géologique et humaine de la Méditerranée.

Ce qui est certain et que l'analyse des données montre, c'est que ce voisinage influe sur l'expérience quotidienne de la Villa. En effet, les deux faits qui ressortent concernant ces deux équipements culturels sont d'une part la rivalité et d'autre part la complémentarité/traitement égale. La rivalité va dans les deux sens, l'un favorable au MuCEM, l'autre favorable à la Villa, le premier étant le plus significatif à notre sens car touchant les aspects essentiels de la Villa. Ainsi, on observe l'effet négatif sur la Villa de la référence au côté doublon et une remise en question de sa légitimité par rapport à son coût, sa raison d'être ou l'espace occupé sur le J4:

\footnotetext{
${ }^{78}$ Cette phrase prononcée par Michel Vauzelle est recueillie par le journaliste lors de l'inauguration officielle.
} 
C'est SCANDALEUX d'avoir sur fonds publics pas réussi à construire un unique bâtiment. Quand on pense au coût de construction et de fonctionnement! Vive le MuCEM! Que faites-vous là?

C'est du gaspillage. On ne comprend pas ce bâtiment. Mauvais emplacement à côté du MuCEM.

Par contre, les visiteurs ayant exprimé un jugement sur cette proximité dans le sens positif pour la Villa mettent en avant l'accueil et l'ambiance:

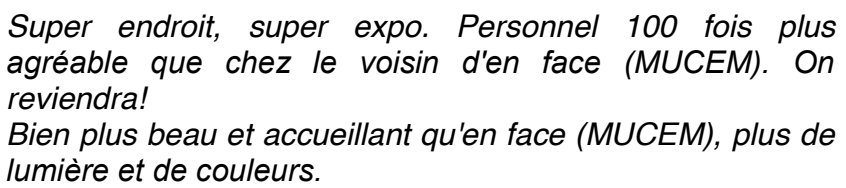

Un autre élément peut ici prolonger les avis mitigés ; il concerne les visiteurs qui s'attendent à voir des peintures et des sculptures. Si l'on prend en compte le fait que la plupart des visiteurs venus à la Villa ne savaient pas ce qu'elle était, on peut se demander dans quelle mesure ces attentes sont dues à ce voisinage avec le MuCEM. Un point frappant qui est observable est l'évidente confusion entre les deux bâtiments. En effet, il y a des visiteurs dont les commentaires dans le Livre d'or ou sur des post-it laissent croire qu'ils pensaient être au MuCEM. Cette confusion semble être due à l'absence de signalétique sur le J4 et pendant des mois sur le bâtiment de la Villa également. En témoigne cet extrait de l'entretien avec un agent d'accueil :

Déjà, il y a un grand souci je pense sur l'extérieur pour l'orientation. Sur la Villa, sur le MuCEM, sur le Fort St. Jean il y a rien qui est indiqué, les gens arrivent ils sont toujours énervés [...] On ne peut pas gérer quand il y a l'Office de tourisme qui leur donne de mauvaises informations et ils viennent tous ici et ils nous demandent: "Par où on passe? On prend les escalateurs pour passer à la passerelle? ", "Non, la passerelle c'est au MuCEM. ", "Ah, mais on est pas au MuCEM ? ".

Pourtant, plus qu'au problème d'absence de signalétique, ce constat renvoie au manque de communication déjà évoqué et signale le faible effort de positionnement de la Villa par rapport au MuCEM, un positionnement qui irait au-delà de la sphère de la rhétorique et passerait par les actions concrètes de démarcation, avec l'objectif d'éviter les conséquences générées par la présence du MuCEM.

\section{Conclusions}

L'analyse de l'expérience de visite nous oblige à reconnaître de larges zones d'ombre dans le procès d'évaluation et d'interprétation de la part des publics et, dans un même temps, elle nous parle de la perception globale, des fonctions et de l'identité complexe de cette nouvelle offre culturelle marseillaise qui semble ne pas être si insensible et si émancipée par rapport à l'évidente proximité, à la fois spatiale et thématique, avec le MuCEM.

Concernant la relation (une relation complexe, à vrai dire) avec ses publics, il nous semble évident que les glissements terminologiques n'ont pas produit de changements significatifs sur le plan des politiques d'accueil ou des services aux publics, en restant le thème 
des outils de médiation, pour n'en citer qu'un, un parmi les plus touchés par les critiques des visiteurs. Ce qui semble véritablement faire trait d'union entre la Villa Méditerranée et ses publics, c'est cette sorte d'appel à l'engagement que suscitent les «parcours ». En ce sens, ce sont de véritables voies d'accès aux émotions des visiteurs: les «parcours » se montrent ainsi capables de toucher leurs valeurs et de les amener vers un voyage à destination des " grands enjeux de la Méditerranée contemporaine " (Dossier de présentation, 2013), tel qu'on l'envisage depuis le discours institutionnel.

A partir de ce constat, la définition de la mission et des fonctions de la Villa demeure un champ délicat. D'un côté, la multiplication des fonctions n'a pas facilité le travail de communication, dont l'insuffisance, voir l'absence, semble être à l'origine de toutes sortes de malentendus avec les visiteurs ${ }^{79}$ appelés à recourir à leurs bagages d'expérience de fréquentation et habitus culturels pour combler ce vide. De l'autre côté, le positionnement de l'établissement de la Villa Méditerranée dans la ville de Marseille en relation avec les autres équipements culturels marseillais (principalement le MuCEM) dans le contexte des politiques culturelles locales n'a pas encore trouvé de solution ${ }^{80}$. II convient de voir si ce n'est pas dans la redéfinition des ambitions institutionnelles que l'identité à venir de la Villa Méditerranée doit se (re)construire.

\section{Remerciements}

Nous tenons à remercier tous les professionnels de la Villa Méditerranée qui, par leur disponibilité et leur engagement, ont accepté de participer à cette recherche en mettant à notre disposition la documentation, mais surtout leur temps et leurs expériences. Enfin, nous remercions tout particulièrement Sylvia Girel pour son soutien et ses conseils sans lesquels ce travail n'aurait jamais vu le jour.

\section{Références}

Ballé, C., \& Poulot, D. (1995). «Les Politiques de public dans les musées européens. " Publics et Musées, 8 (1), 124-131.

Béra, M.-P., \& Paris, E. (2007). " Usages et enjeux de l'analyse des livres d'or pour les stratégies culturelles d'établissement. " Dans Eidelman, J., Roustan, M. \& Goldstein, B. (Eds.). (2007). La place des publics. De l'usage des études et recherches par les musées. (pp. 199-211). Paris : La Documentation française.

Buslacchi, M.-E., Girel, S., \& Maisetti, N. (2015). "Le Musée des Civilisations de l'Europe et de la Méditerranée (MuCEM) à Marseille : succès et controverses autour d'un équipement culturel de développement territorial. " Dans G. Baudelle, G. Krauss, \& J.-F. Polo (Eds.). Musées d'art et développement territorial. Rennes: Presses Universitaires de Rennes.

Chaumier, S. (2007). "Le public, acteur de la production d'exposition ? Un modèle écartelé entre enthousiasme et réticenses. " Dans J. Eidelman, M. Roustan, \& B. Goldstein (Eds.). (2007). La place des

\footnotetext{
${ }^{79}$ Les changements introduits dans la gestion du lieu et, notamment, avec l'extension de l'entrée libre à tous les parcours, l'affichage de la programmation de façon très visible sur les baies vitrées du bâtiment, indiquent pourtant que les doléances des publics ne sont pas restées sans auditoire.

${ }^{80}$ Au moment où nous rendons cet article, le statut de la Villa change et vient poser de nouvelles questions sur son avenir. En effet, l'année 2015 signale le passage au statut de GIP AViTeM (Groupement d'intérêt public Agence des villes et territoires durables méditerranéens), http://www.villa-mediterranee.org/fr/enjeux-et-projets-2015
} 
publics. De l'usage des études et recherches par les musées. (pp. 241-250). Paris : La Documentation française.

Davallon, J. (1992). I « ntroduction. Le public au centre de l'évolution du musée. " Publics et Musées, 2 (1), 10-18.

Davallon, J. (1997). L'évolution du rôle des musées. " La lettre de IOCIM, 49, 4-8.

Villa Méditerranée (2013). Dossier de présentation.

Dufresne-Tassé, C. (2002). " L'évaluation, recherche appliquée aux multiples usages. " Paris, ICOM CECA. Paris: ICOM CECA.

Eidelman, J., Roustan, M., \& Goldstein, B. (Eds.). (2007). La place des publics. De l'usage des études et recherches par les musées. Paris : La Documentation française.

Eidelman, J., \& Céroux, B. (2009). "La gratuité dans les musées et monuments en France : quelques indicateurs de mobilisation des visiteurs. " Culture Etudes -Pratiques et Publics, 2, 1996-2000.

Eidelman, J., \& Jonchery, A. (2013). " A I' écoute des visiteurs 2012 »: résultats de l'enquête nationale sur la satisfaction des publics des musées nationaux.Département de la politique des publics, DGPMCC,1-14.

Freschel, A. (2013, juin 18). "La contestée - à tort ? - Villa Méditerranée. » Journal Zibeline. Consulté à partir de http://www.journalzibeline.fr/culture-politique/.

Freschel, A. \& Cloarec, G. (2012, novembre 16). « La Villa Méditerranée va naître. Entretien avec François de Boisgelin, Directeur de la Villa Méditerranée. » Zibeline. Consulté à partir de http://www.journalzibeline.fr/la-villa-mediterranee-va-naitre/.

Gilles, B. (2013, avril 6). "Villa Méditerranée : "Il y a un côté fou dans ce bâtiment". " Marsactu. Consulté à partir de http://www.marsactu.fr/archi-et-urbanisme/villa-mediterranee-il-y-aun-cote-fou-dans-ce-batiment-30801.html.

Girel, S. (2013). « Marseille Provence 2013 - Publics et pratiques culturelles dans une capitale européenne de la culture. Un programme de recherche et des dispositifs d'enquêtes. " Retrieved from http://mp2013publicspratiques .wordpress.com/about/.

Jauss, H. R. (1990). Pour une esthétique de la réception. Paris : Gallimard.

Jordi, J.-J. (2013). « Un site emblématique. » TDC, 1055, 16-19.

La Villa Méditerranée, enjeux et projets 2015. (s.d.). Villa Méditerranée. Retrieved from http://www.villa-mediterranee.org/fr/enjeux-et-projets2015.

L'architecture. "La Villa Méditerranée un bâtiment entre ciel et mer. » (s.d.). Villa Méditerranée. Consulté à partir de http://www.villamediterranee.org/fr/la-villa-mediterranee-un-batiment-entre-ciel-etmer.

Leforestier, J.-M. (2013, janvier 11). « Michel Vauzelle reçoit en sa villa. » Marsactu. Consulté à partir de http://www.marsactu.fr/politique/michel-vauzelle-recoit-en-sa-villa30010.html.

Le Marec, J. (2007). «Muséologie particiative, évaluation, prise en compte des publics : la parole introuvable. " In Eidelman, J., Roustan, M. \& Goldstein, B. (Eds.). La place des publics. De l'usage des études et recherches par les musées. (pp. 251-268). Paris : La Documentation française.

Le projet. "S'emparer des grands enjeux de la Méditerranée. » (s.d.) Villa Méditerranée. Consulté à partir de http://www.villamediterranee.org/fr/semparer-des-grands-enjeux-de-lamediterranee-contemporaine.

Mairesse, F. (2010). Le musée hybride. Paris: La Documentation française.

Oberson, F. (2013, octobre 30). "La villa du seigneur de PACA » [Blog post]. Consulté à partir de http://blogs.mediapart.fr/edition/visages-demarseille/article/301013/la-villa-du-seigneur-de-paca.

Piscopo-Reguieg, S. (2013, Avril 11). « Marseille-Provence 2013 : la Villa Méditerranée cherche sa place. "Télérama. Consulté à partir de http://www.telerama.fr/scenes/marseille-provence-2013-la-villamediterranee-cherche-sa-place,96069.php.

Poirrier, P. (1996). "Changements de paradigmes dans les politiques culturelles des villes. " Hermès, (20), 85. 
Savane, M. (2013, mai 8). « Marseille, Villa Méditerranée : innocent plagiat architectural? » César. Consulté à partir de http://www.cesar.fr/plagiat-villa-mediterranee-marseille-2013.

Simon, N. (2010). The participatory museum. Santa Cruz: Museum 2.0.

Tobelem, J.-M. (2003). "Utilisation des études de publics et straégies de développement des organisations culturelles. " Dans O. Donnat, \& P. Tolila (Eds.). (2003). Le(s) public(s) de la culture- Vol. II (cédérom) (pp. 251-259). Paris : Presses de Scieces Po.

Tobelem, J.-M. (2010). Le nouvel âge des musées. Les institutions culturelles au défi de la gestion. Paris: Armand Colin.

Tobelem, J.-M., \& Benito, L. (2001). «Les musées dans la politique touristique urbaine. " Politique et Musée (pp. 263-293). Paris : L'Harmattan.

\section{Résumé}

La Villa Méditerranée de Marseille a été ouverte au public en juin 2013 avec l'inauguration de deux "parcours " d'exposition. Depuis sa genèse, l'effort de ce nouvel équipement culturel semble être marqué par une volonté réformatrice et pionnière, soit dans le domaine des contenus proposés, soit dans la façon dont ces contenus sont amenés à être communiqués aux publics : déjà dans le discours institutionnel l'exposition se fait "parcours", le commissaire "narrateur », le visiteur « acteur ». Quelles sont alors les conséquences de ce glissement terminologique dans l'expérience quotidienne de l'institution ? À travers l'usage combiné des entretiens semi-directifs, d'une observation participante et de l'interprétation du Livre d'Or, cette étude vise à dépasser les limites des définitions fermées au service d'une perspective critique qui observe la relation entre les attentes institutionnelles et l'évidence de la réception des "parcours ». L'analyse de l'expérience de visite nous amène à reconnaître des zones d'ombre dans le procès d'évaluation et d'interprétation de la part des publics et, dans un même temps, elle nous parle de la perception globale de la Villa Méditerranée qui semble ne pas être si insensible qu'il y paraît à l'évidente proximité, à la fois spatiale et thématique, avec le Musée des Civilisations de l'Europe et de la Méditerranée (MuCEM).

Mots clé : parcours des visiteurs, étude des visiteurs, musée des civilisations

\section{Abstract}

New trends, old problems? The case for reception of the " pathway » at the Ville Méditerranée in Marseille

The Villa Méditerranée in Marseille opened to visitors in June 2013 with the inauguration of two exhibitions. Since the beginning, this new cultural institution resolutely points toward a new way of constructing and communicating exhibits. Exhibits become "pathways", curators become "storytellers", visitors are "actors". What are the practical and theoretical consequences of this shift in terminology? Through the use of both semi-structured interviews and notes in the visitors' book, this study aims to cross the boundaries of standard definitions and find the relation between institutional expectations and visitors' actual experiences. Visitors' reactions allowed us to recognize some blind areas in the evaluation process and interpretation of institutional proposals and, at the same time, they made us aware of some characteristics of the Villa Méditerranée. For example, the physical and thematic proximity of the Musée des Civilisations de l'Europe et de la Mediterranée (MuCEM) appears to influence the visit in the Villa Méditerranée.

Key words: visitor route, visitor studies, museums of civilizations 


\section{Resumen}

Nuevas tendencias, antiguos problemas? El caso de la recepción de los "parcours" de la Villa Méditerranée de Marseilla

La Villa Méditerranée de Marsella abrió sus puertas el pasado junio 2013 presentando a sus públicos dos « recorridos » expositivos. Desde el principio, el esfuerzo de la nueva institución cultural ha sido fuertemente marcado por cierto afán renovador, tanto por lo que se refiere a la oferta de los contenidos, como en la manera de comunicarse a sus públicos. Ya desde el discurso oficial, la exposición se hace "recorrido", el comisario " narrador", el visitante «actor». ¿Cuáles son las consecuencias de estos cambios terminológicos en la experiencia diaria de la institución? Gracias al uso conjunto de entrevistas semi-directivas, de una observación participante y de la interpretación de las notas del Libro de los Visitantes, con este estudio nos proponemos ir más allá de las definiciones cerradas adoptando una perspectiva crítica que nos permita observar a la vez la relación entre las expectativas institucionales y las evidencias de la recepción de los "recorridos". El análisis de la experiencia de visita revela algunos puntos críticos en el proceso de evaluación e interpretación de parte de los públicos y al mismo tiempo nos habla de la percepción global de la Villa Méditerranée, la cual acaba padeciendo la evidente cercanía, tanto semántica como espacial, con el astro naciente del MuCEM (Musée des Civilisations de l'Europe et de la Mediterranée).

Palabras-clave: estudio de visitantes, recorrido del visitante, museos de las civilizaciones 